\title{
HIGHLIGHTS
}

STEM CELLS

\section{MSCs can inhibit T-cell proliferation in vitro but not in vivo}

Researchers in Belgium have uncovered some unexpected findings: mesenchymal stem cells (MSCs) can potently inhibit $\mathrm{T}$-cell proliferation in an interferon (IFN)- $\gamma$-dependent manner in vitro, but have no effect on T-cell proliferation or the development of collagen-induced arthritis (CIA) in vivo.

The team of investigators, led by Patrick Matthys, previously showed that regulatory $\mathrm{T}$ cells ( $\mathrm{T}_{\mathrm{REG}}$ cells) inhibited T-cell activation in vitro and that these cells could prevent the development of CIA in mice. Encouraged by these results, the researchers generated MSCs, which have been reported to show immunosuppressive and regenerative activities. In vitro, wild-type MSCs inhibited T-cell proliferation (induced by anti-CD3 antibodies) in a dose-dependent manner. By contrast, MSCs isolated from mice lacking the IFN- $\gamma$ receptor showed a considerably lower inhibitory capacity, indicating the importance of IFN- $\gamma$ signaling in MSCs in the suppression of T-cell proliferation.

So, which molecules are involved in this suppression? In response to stimulation (by interleukin-17 and IFN- $\gamma$ ), the expression of inducible nitric oxide synthase (iNOS),

programmed death ligand-1 (PD-L1) and cyclooxygenase (COX)2, but not indoleamine 2,3-dioxygenase (IDO), was upregulated in MSCs, indicating their potential involvement in mediating T-cell inhibition. The abrogation of T-cell

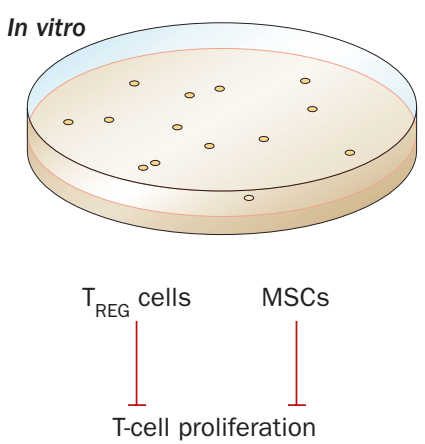

proliferation by inhibitors of iNOS and COX2 confirmed the involvement of these molecules in the process.

These positive findings prompted Matthys and colleagues to investigate whether MSCs could counteract the development of CIA, similar to their findings using $\mathrm{T}_{\text {REG }}$ cells. Following immunization with collagen II, mice were injected once with $10^{6}$ MSCs. "Regrettably, MSCs were not able to inhibit the development of CIA," states Matthys. In the next four experiments we then changed the route, the number and time points of MSC injections but none of the treatments had a beneficial effect on |the development of arthritic symptoms, and didn't induce changes in the accompanying cellular and humoral autoimmune responses nor on the proliferation of $\mathrm{T}$ cells in vivo. Thus, unlike $\mathrm{T}_{\mathrm{REG}}$ cells, the immunomodulatory properties of murine MSCs, as evident from in vitro experiments, cannot be extrapolated towards an in vivo model, such as CIA."

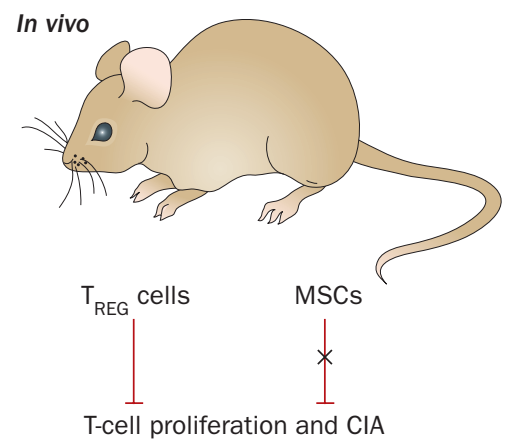

It is worth noting that, as outlined in the discussion of the report by Matthys' group, two out of five other similar studies reported no beneficial effects of MSCs on arthritic symptoms. In the three studies that showed less-severe disease symptoms following MSC treatment, higher doses of MSCs were used, or the MSCs were derived from human or mouse adipose tissue, rather than bone marrow. Another possibility is that intravenously injected MSCs cannot inhibit T-cell proliferation and CIA because they might not reach the spleen and lymph nodes; MSCs have been reported to lose their capacity to home to lymphoid organs after a short time in culture. The advantage of MSCs, though, is their potential to be genetically manipulated to home to inflamed tissues.

\section{Katrin Legg}

Original article Schurgers, E. et al. Discrepancy between the in vitro and in vivo effects of murine mesenchymal stem cells on T-cell proliferation and collagen-induced arthritis. Arthritis Res. Ther. 12, R31 (2010) 